\title{
Optimal Threshold Value of Digital Dynamic System
}

\author{
Ful-Chiang $\mathrm{Wu}^{1}{ }^{*}$, Thomas C. Chuang², Ya-Chi Lee ${ }^{1}$, Ruei-Lung Lai ${ }^{3}$ \\ ${ }_{1}^{1}$ Department of Hotel Management, Vanung University, Tao-Yuan 32601, Taiwan. \\ 2 Department of Information Science and Technology, Vanung University, Tao-Yuan 32601, Taiwan. \\ ${ }^{3}$ Department of Tourism and Leisure Management, Vanung University, Tao-Yuan 32601, Taiwan. \\ * Corresponding author. Tel.: +886-3-4515811\#86603; email: shogo@vnu.edu.tw \\ Manuscript submitted September 27, 2018; accepted March 1, 2019. \\ doi: 10.17706/ijcee.2019.11.2.101-107
}

\begin{abstract}
Taguchi digital dynamic system are problems in which both the input signal and the output responses have values of either 0 or 1 with two misclassification probabilities. Taguchi proposed a two-step procedure for the digital dynamic system under an equalized error rates model and maximized the standardized SN ratio to achieve robust design. This paper proposes a quality loss model to determine the optimal threshold value of a digital dynamic system based on normally distributed data with unequal loss coefficients. We varied the variances of random variable $\mathrm{X}_{1}$ with increment of 0.10 using Excel software. The results are compared with the threshold values obtained by using Taguchi method. When the two loss coefficients and variances of two populations are equal, the optimal threshold value is the same as the threshold value provided by Taguchi method. The maximum error of optimal threshold value is 0.03 compared with actual threshold value.
\end{abstract}

Key words: Digital dynamic system, error rate, Taguchi method, threshold value.

\section{Introduction}

The Taguchi method [1] has been widely used successfully in various fields to optimize the design problems, which can be classified into static and dynamic problems depending upon the absence or presence of signal factors, respectively. The desired output of static system has a fixed target value and the desired output of dynamic system varies depending on the level of the signal factor set. Signal-to-noise (SN) ratio is proposed by Taguchi to be used to optimize the parameter settings, which is a statistic derived from the quality loss function. A higher SN ratio means a lower loss.

The dynamic systems can be divided into four classes: (1) Continuous-continuous; (2) Continuous-digital; (3) Digital-continuous; and (4) Digital-digital. In case of the digital dynamic system, both input signal and output response have values of either 0 or 1 with two misclassification probabilities occurring.

Many researchers have actively worked on the robust design of dynamic systems. Kapur and Chen [2] provided the equations for computing the SN ratio for either equispaced or non-equispaced levels for the signal factors. Phadke and Dehnad [3] presented a two-step optimization procedure independent of the chosen distribution. Miller and $\mathrm{Wu}$ [4] derived the response function modeling approach for a dynamic system. Wasserman [5] demonstrated Taguchi SN ratio with a linear statistical model. Lunani, Nair and Wasserman [6] developed two graphical methods for identifying appropriate measures of location and dispersion for dynamic experimental designs. McCaskey and Tsui [7] proposed a two-step procedure for dynamic systems under an additive model to reduce the dimension of the optimization problem. Su and 
Hsieh [8] used the neural network technique to optimize the robust design of dynamic system. Tsui [9] compared the effect estimates obtained using Taguchi loss model and response model approach for dynamic system. Li [10] established three models for achieving robust design of digital dynamic system by selecting the optimal threshold value. Chen [11] used a stochastic sequential quadratic programming to achieve robust design for dynamic systems. Wu, Wang and Fan [12] proposed an approach to obtain the cut-off point by minimizing quality loss for the digital dynamic systems where the output is classified into four classes under normally distributed data. Al-Refaie et al. [13] integrated the desirability function and data envelopment analysis for solving dynamic systems with multi-responses. Gauri and Pal [14] proposed a multiple regression-based weighted signal-to-noise ratio (MRWSN) to optimize the multi-response dynamic systems.

This paper develops a quality loss model to determine optimal threshold value for a digital dynamic system. The loss coefficients of error rates $\mathrm{p}$ and $\mathrm{q}$ in digital dynamic system are not equal.

\section{Digital Dynamic System}

The outcomes of a binary classification model for the digital dynamic system are labeled either as $X_{0}$ and $X_{1}$. The ideal function of digital dynamic system is that whenever an input signal is $X_{0}$, the output should be $X_{0}$, and whenever the input signal is $X_{1}$, the output should be $X_{1}$. The accurate outcomes for input signals $X_{0}$ and $X_{1}$ are $n_{00}$ and $n_{11}$ respectively, and the wrong outcomes for input signals $X_{0}$ and $X_{1}$ are $n_{01}$ and $n_{10}$ respectively. Hence, the number of responses for output signals $X_{0}$ and $X_{1}$ are $r_{0}\left(r_{0}=n_{00}+n_{10}\right)$ and $r_{1}\left(r_{1}=\right.$ $\left.n_{01}+n_{11}\right)$ respectively. The observed frequencies of data can be tabulated the contingency table for a dynamic system as shown in Table 1.

Table 1. Input/Output Table for Digital Dynamic Systems

\begin{tabular}{c|c|c|c}
\hline Input & $X_{0}$ & $X_{1}$ & Total \\
\hline$X_{0}$ & $n_{00}$ & $n_{01}$ & $n_{0}$ \\
\hline$X_{1}$ & $n_{10}$ & $n_{11}$ & $n_{1}$ \\
\hline Total & $r_{0}$ & $r_{1}$ & $n$ \\
\hline \hline
\end{tabular}

Let the output be a continuous random variable affected by control factors $\mathbf{Z}$ and noise factors $\mathbf{N}$. The threshold value $R$ critically affects the judgement of output. If the output values, that are smaller than or equal to threshold $R$, are set as $X_{0}$, otherwise they are $X_{1}$. Fig. 1 shows the criterion of threshold $R$ of digital dynamic system.

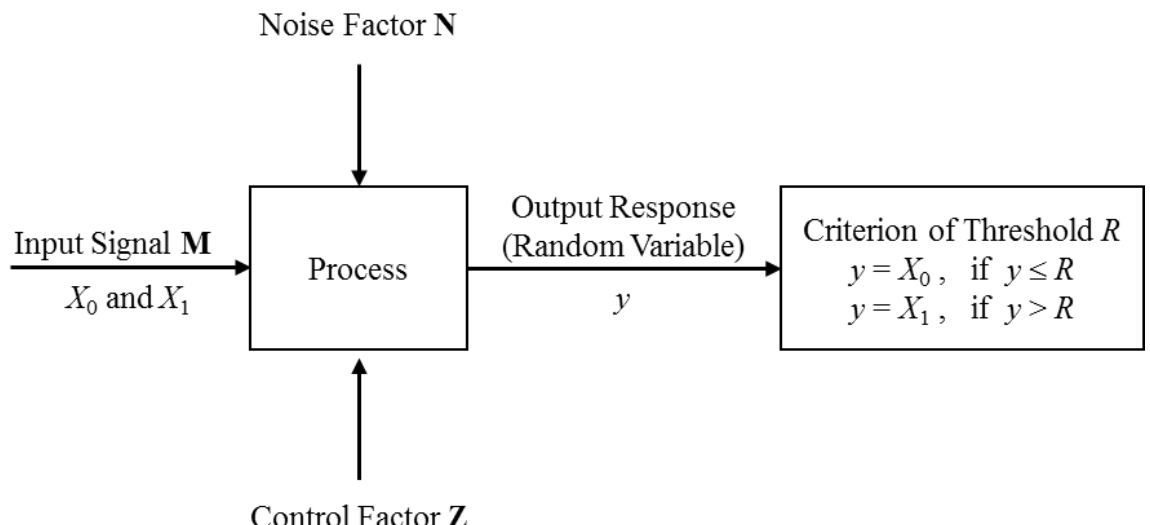

Fig. 1. The model of digital dynamic system. 
Let $f(y \mid X)$ represent the function of output $y$ given input signal $M=X_{0}$ or $M=X_{1}$. The error rate $p(=$ $\left.n_{01} / n_{0}\right)$ presents the probability of wrongly classifying signal $X_{0}$ as $X_{1}$, and $q\left(=n_{10} / n_{1}\right)$ presents the probability of wrongly classifying signal $X_{1}$ as $X_{0}$. The data in Table 1 can be converted into those in Table 2 .

Table 2. Input/Output Table in Terms of Error Rate

\begin{tabular}{c|c|c|c}
\hline \hline Onput & $X_{0}$ & $X_{1}$ & Total \\
\hline$X_{0}$ & $1-p$ & $p$ & 1 \\
\hline$X_{1}$ & $q$ & $1-q$ & 1 \\
\hline Total & $1-p+q$ & $1+p-q$ & 2 \\
\hline
\end{tabular}

Obviously, error rates $p$ and $q$ are inversely related and vary with the threshold $R$. Moving the threshold $R$ to the left decreases the error rate $q$ but also increases the error rate $p$. Vice versa, moving the threshold $R$ to the right decreases the error rate $p$ but also increases the error rate $q$. This means that $p$ and $q$ cannot be increased concomitantly. The value of threshold $R$ is selected so that there is a trade-off between $p$ and $q$. Fig. 2 shows the threshold $R$ chosen to determine the error rates $p$ and $q$.

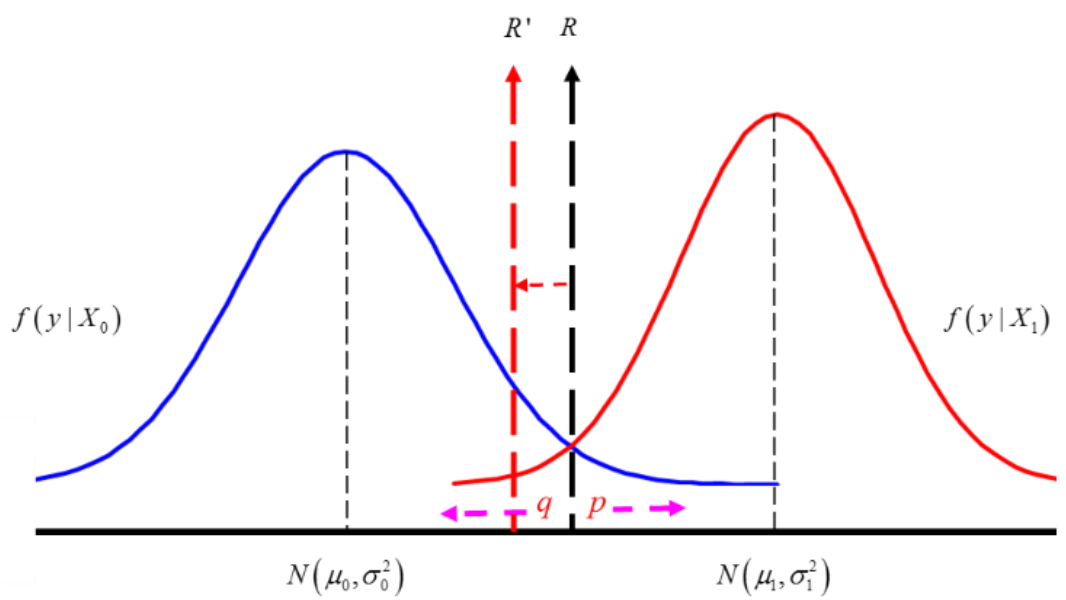

Fig. 2. Adjustment of the threshold $R$ alters the $p$ and $q$.

When the parameters of bi-normal distribution of output responses were unknown, Taguchi [1] used the following formula to calculate the equalized error rate based on the two error rates $p$ and $q$ with the same loss coefficient, which is denoted by $p_{0}$.

$$
p_{0}=[1+\sqrt{(1 / p-1)(1 / q-1)}]^{-1}
$$

\section{The Threshold Value of Digital Dynamic System}

The relationships between $p$ and $q$ depend on the distributions of output responses. Let the random variables $X_{0}$ and $X_{1}$ represent the results of output responses having independent normal distributions $X_{0} \sim N\left(\mu_{0}, \sigma_{0}^{2}\right)$, and $X_{1} \sim N\left(\mu_{1}, \sigma_{1}^{2}\right)$ respectively, and assume that $\mu_{1}>\mu_{0}$. The sensitivity and specificity are presented as

$$
p=1-\Phi\left(\left(R-\mu_{0}\right) / \sigma_{0}\right)
$$




$$
q=\Phi\left(\left(R-\mu_{1}\right) / \sigma_{1}\right)
$$

where $\Phi(\cdot)$ denotes the cumulative distribution function for the standard normal distribution.

According to (1), the threshold $R_{T}$ of Taguchi method is obtained by

$$
R_{T}=\frac{\mu_{D} \sigma_{H}+\mu_{H} \sigma_{D}}{\sigma_{D}+\sigma_{H}}
$$

To obtain one accurate output $y=X_{0}$, on average, we have to obtain $p /(1-p)$ pieces wrong output $y=X_{1}$ when input signal $M=X_{0}$. Let $K_{1}$ be the cost of processing one accurate output for input signal as $X_{0}$. The quality loss $L_{1}$ is

$$
L_{1}(R)=K_{1} \cdot \frac{p}{1-p}=K_{1} \cdot\left[\frac{1}{\Phi\left(\left(R-\mu_{0}\right) / \sigma_{0}\right)}-1\right]
$$

Similarly, suppose that $K_{2}$ is the cost of processing one accurate output for input signal as $X_{1}$, the quality loss $L_{2}$ is given by

$$
L_{2}(R)=K_{2} \cdot \frac{q}{1-q}=K_{2} \cdot\left[\frac{1}{1-\Phi\left(\left(R-\mu_{1}\right) / \sigma_{1}\right)}-1\right]
$$

Hence, the total quality loss $(L)$ can be expressed as

$$
L(R)=L_{1}(R)+L_{2}(R)
$$

The threshold $R$ that achieves this minimum quality loss will be considered as the optimal threshold value $R^{*}$.

$$
R^{*}=\min _{R}[L(R)]=\min _{R}\left\{K_{1} \cdot \Phi\left(\left(R-\mu_{0}\right) / \sigma_{0}\right)^{-1}+K_{2} \cdot\left[1-\Phi\left(\left(R-\mu_{1}\right) / \sigma_{1}\right)\right]^{-1}\right\}
$$

Differentiating $L(R)$ with respect to $R$ and then setting the derivative be equal to zero, the optimal threshold value $R^{*}$ is obtained. Hector et al. [15] proposed a high accurate simple approximation of normal distribution integral, which is expressed as

$$
\Phi(x) \cong\left(1+\exp \left(-\frac{358}{23} x+111 \arctan \left(\frac{37}{294} x\right)\right)\right)^{-1}
$$

Regrettably, there is no closed-form solution available for (9).

Bowling et al. [16] provided a logistic approximation function to the cumulative normal distribution, which is expressed as 


$$
\Phi(x) \cong(1+\exp (-1.702 \cdot x))^{-1}
$$

Therefore, using (10), the optimal threshold value $R^{*}$ is given by

$$
R^{*}=\left(1.702\left(\mu_{D} \sigma_{H}+\mu_{H} \sigma_{D}\right)+\sigma_{D} \sigma_{H} \ln \left(\left(K_{1} \cdot \sigma_{\mathrm{D}}\right) /\left(K_{2} \cdot \sigma_{\mathrm{H}}\right)\right)\right) /\left(1.702\left(\sigma_{D}+\sigma_{H}\right)\right)
$$

When $K_{1}$ is equal to $K_{2}$ and $\sigma_{H}=\sigma_{D},(11)$ is rewritten as

$$
R^{*}=\frac{\mu_{H}+\mu_{D}}{2}
$$

In this study, we propose a mixture model of (9) and (11) to calculate the optimal threshold value $R^{*}$. The formula is expressed as

$$
R^{*}=\left(A \cdot\left(\mu_{D} \sigma_{H}+\mu_{H} \sigma_{D}\right)+\sigma_{D} \sigma_{H} \ln \left(\left(K_{1} \cdot \sigma_{\mathrm{D}}\right) /\left(K_{2} \cdot \sigma_{\mathrm{H}}\right)\right)\right) /\left(A \cdot\left(\sigma_{D}+\sigma_{H}\right)\right)
$$

where $\mathrm{A}$ is

$$
A=1.08206 \cdot \arctan \left(3.60102 \cdot\left(\mu_{D}-\mu_{H}\right)+2.53909\right)
$$

\section{Analysis of Optimal Threshold Values}

We analyzed the pairs of bi-normal distributions with equal and unequal variances. The distribution of $X_{0}$ included standard normal distribution as $N\left(0,1^{2}\right)$ and $X_{1}$ as $N\left(1, \sigma_{1}^{2}\right)$ and $N\left(2, \sigma_{1}^{2}\right)$. In one pair, ratio of cost coefficients $K_{1}: K_{2}$ was assigned as $K_{1}: K_{2}=1: 1$ and $K_{1}: K_{2}=1: 2$. The optimal threshold value derived for the Taguchi method and proposed method are listed in Table 3 and Table 4.

Table 3. The Threshold Values from Different Methods $\left(X_{0}: N\left(0,1^{2}\right), X_{1}: N\left(1, \sigma_{1}^{2}\right)\right)$

\begin{tabular}{c|c|c|c|c|c|c|c|c}
\hline \hline & \multicolumn{5}{|c|}{$K_{1}: K_{2}=1: 1$} & \multicolumn{3}{c}{$K_{1}: K_{2}=1: 2$} \\
\hline$\sigma_{1}$ & Actual value & $\begin{array}{c}\text { Taguchi } \\
\text { method }\end{array}$ & $\begin{array}{c}\text { Bowling } \\
\text { model }\end{array}$ & $\begin{array}{c}\text { Proposed } \\
\text { method }\end{array}$ & Actual value & $\begin{array}{c}\text { Taguchi } \\
\text { method }\end{array}$ & $\begin{array}{c}\text { Bowling } \\
\text { model }\end{array}$ & $\begin{array}{c}\text { Proposed } \\
\text { method }\end{array}$ \\
\hline 0.50 & 0.51593 & 0.66667 & 0.53092 & 0.51516 & 0.36978 & 0.66667 & 0.39516 & 0.36365 \\
0.60 & 0.49931 & 0.62500 & 0.51245 & 0.49939 & 0.33191 & 0.62500 & 0.35973 & 0.32894 \\
0.70 & 0.49160 & 0.58824 & 0.50194 & 0.49193 & 0.30595 & 0.58824 & 0.33425 & 0.30477 \\
0.80 & 0.49023 & 0.55556 & 0.49729 & 0.49052 & 0.28875 & 0.55556 & 0.31628 & 0.28851 \\
0.90 & 0.49344 & 0.52632 & 0.49699 & 0.49359 & 0.27811 & 0.52632 & 0.30408 & 0.27829 \\
1.00 & 0.50000 & 0.50000 & 0.50000 & 0.50000 & 0.27244 & 0.50000 & 0.29637 & 0.27274 \\
1.10 & 0.50905 & 0.47619 & 0.50552 & 0.50893 & 0.27057 & 0.47619 & 0.29220 & 0.27084 \\
1.20 & 0.51996 & 0.45455 & 0.51298 & 0.51976 & 0.27165 & 0.45455 & 0.29084 & 0.27184 \\
1.30 & 0.53226 & 0.43478 & 0.52191 & 0.53202 & 0.27504 & 0.43478 & 0.29172 & 0.27512 \\
1.40 & 0.54560 & 0.41667 & 0.53199 & 0.54537 & 0.28025 & 0.41667 & 0.29442 & 0.28023 \\
1.50 & 0.55970 & 0.40000 & 0.54294 & 0.55953 & 0.28690 & 0.40000 & 0.29858 & 0.28681 \\
1.60 & 0.57436 & 0.38462 & 0.55455 & 0.57428 & 0.29469 & 0.38462 & 0.30393 & 0.29457 \\
1.70 & 0.58942 & 0.37037 & 0.56667 & 0.58945 & 0.30340 & 0.37037 & 0.31025 & 0.30327 \\
1.80 & 0.60475 & 0.35714 & 0.57915 & 0.60492 & 0.31284 & 0.35714 & 0.31735 & 0.31273 \\
1.90 & 0.62025 & 0.34483 & 0.59190 & 0.62058 & 0.32286 & 0.34483 & 0.32508 & 0.32279 \\
2.00 & 0.63584 & 0.33333 & 0.60484 & 0.63635 & 0.33333 & 0.33333 & 0.33333 & 0.33333 \\
\hline \hline
\end{tabular}


Table 4. The Threshold Values from Different Methods $\left(X_{0}: N\left(0,1^{2}\right), X_{1}: N\left(2, \sigma_{1}^{2}\right)\right)$

\begin{tabular}{c|c|c|c|c|c|c|c|c}
\hline \hline & \multicolumn{5}{|c|}{$K_{1}: K_{2}=1: 1$} & & \multicolumn{2}{c}{$K_{1}: K_{2}=1: 2$} \\
\hline$\sigma_{1}$ & Actual value & $\begin{array}{c}\text { Taguchi } \\
\text { method }\end{array}$ & $\begin{array}{c}\text { Bowling } \\
\text { model }\end{array}$ & $\begin{array}{c}\text { Proposed } \\
\text { method }\end{array}$ & Actual value & $\begin{array}{c}\text { Taguchi } \\
\text { method }\end{array}$ & $\begin{array}{c}\text { Bowling } \\
\text { model }\end{array}$ & $\begin{array}{c}\text { Proposed } \\
\text { method }\end{array}$ \\
\hline 0.50 & 1.19974 & 1.33333 & 1.19758 & 1.18793 & 1.07253 & 1.33333 & 1.06183 & 1.04252 \\
0.60 & 1.13574 & 1.25000 & 1.13745 & 1.12945 & 0.98567 & 1.25000 & 0.98473 & 0.96587 \\
0.70 & 1.08683 & 1.17647 & 1.09018 & 1.08404 & 0.91621 & 1.17647 & 0.92249 & 0.90443 \\
0.80 & 1.04954 & 1.11111 & 1.05284 & 1.04870 & 0.86064 & 1.11111 & 0.87184 & 0.85482 \\
0.90 & 1.02125 & 1.05263 & 1.02331 & 1.02122 & 0.81618 & 1.05263 & 0.83040 & 0.81460 \\
1.00 & 1.00000 & 1.00000 & 1.00000 & 1.00000 & 0.78064 & 1.00000 & 0.79637 & 0.78189 \\
1.10 & 0.98430 & 0.95238 & 0.98171 & 0.98380 & 0.75229 & 0.95238 & 0.76839 & 0.75531 \\
1.20 & 0.97302 & 0.90909 & 0.96752 & 0.97168 & 0.72976 & 0.90909 & 0.74538 & 0.73374 \\
1.30 & 0.96529 & 0.86957 & 0.95669 & 0.96289 & 0.71198 & 0.86957 & 0.72651 & 0.71633 \\
1.40 & 0.96043 & 0.83333 & 0.94865 & 0.95685 & 0.69810 & 0.83333 & 0.71109 & 0.70240 \\
1.50 & 0.95790 & 0.80000 & 0.94294 & 0.95310 & 0.68743 & 0.80000 & 0.69858 & 0.69137 \\
1.60 & 0.95728 & 0.76923 & 0.93917 & 0.95125 & 0.67945 & 0.76923 & 0.68855 & 0.68281 \\
1.70 & 0.95825 & 0.74074 & 0.93704 & 0.95100 & 0.67371 & 0.74074 & 0.68062 & 0.67634 \\
1.80 & 0.96052 & 0.71429 & 0.93630 & 0.95208 & 0.66985 & 0.71429 & 0.67449 & 0.67166 \\
1.90 & 0.96388 & 0.68966 & 0.93673 & 0.95430 & 0.66758 & 0.68966 & 0.66991 & 0.66851 \\
2.00 & 0.96814 & 0.66667 & 0.93817 & 0.95748 & 0.66667 & 0.66667 & 0.66667 & 0.66667 \\
\hline \hline
\end{tabular}

\section{References}

[1] Taguchi, G. (1992). Taguchi Methods: Research and Development. Dearborn, MI: American Suppliers Institute.

[2] Kapur, K. C., \& Chen, G. (1988). Signal-to-noise ratio development for quality engineering. Quality and Reliability Engineering International, 4(2), 133-141.

[3] Phadke, M. S., \& Dehnad, K. (1988). Optimization of product and process design for quality and cost. Quality and Reliability Engineering International, 4(2), 105-112.

[4] Miller, A., \& Wu, C. F. J. (1996). Commentary on Taguchi's parameter design with dynamic characteristics. Quality and Reliability Engineering International, 12(2), 75-78.

[5] Wasserman, G. S. (1996). Parameter design with dynamic characteristics: A regression perspective. Quality and Reliability Engineering International, 12(2), 113-117.

[6] Lunani, M., Nair, V. N., \& Wasserman, G. S. (1997). Graphical methods for robust design with dynamic characteristics. Journal of Quality Technology, 29(3), 327-338.

[7] McCaskey, S. D., \& Tsui, K. L. (1997). Analysis of dynamic design experiments. International Journal of Production Research, 35(6), 1561-1574.

[8] Su, C. T., \& Hsieh, K. L. (1998). Appling neural network approach to achieve robust design for dynamic quality characteristics. International Journal of Quality and Reliability Management, 15(5), 509-519.

[9] Tsui, K. L. (1999). Modeling and analysis of dynamic robust design experiments. IIE Transactions, 31(12), 1113-1122.

[10] Li, M. H. (2001). Optimising operating conditions by selection of optimal threshold value for digital-digital dynamic characteristic. International Journal of Advanced Manufacturing Technology, 17(3), 210-215.

[11] Chen, S. P. (2003). Robust design with dynamic characteristics using stochastic sequential quadratic programming. Engineering Optimization, 35(1), 79-89.

[12] Wu, F.-C., Wang, H.-M., \& Fan, T.-Y. (2010). Quality design of Taguchi's digital dynamic systems. International Journal of Industrial Engineering - Applications and Practice, 16(5), 405-416.

[13] Al-Refaie, A., Al-Alaween, W., Diabat, A., \& Li, M.-H. (2017). Solving dynamic systems with 
multi-responses by integrating desirability function and data envelopment analysis. Journal of Intelligent Manufacturing, 28(2), 387-403.

[14] Gauri, S. K., \& Pal, S. (2017). Optimization of multi-response dynamic systems using multiple regression-based weighted signal-to-noise ratio. International Journal of Industrial Engineering Computations, 8(1), 161-178.

[15] Hector, V.-L., Roberto, C.-S., Uriel, F.-N., Arturo, S.-R., \& Jesus, S.-O. (2011). High accurate simple approximation of normal distribution integral. Mathematical Problems in Engineering, 2012.

[16] Bowling, S. R., Khasawneh, M. T., Kaewkuekool, S., \& Cho, B. R. (2009). A logistic approximation to the cumulative normal distribution. Journal of Industrial Engineering and Management, 2(1), 114-127.
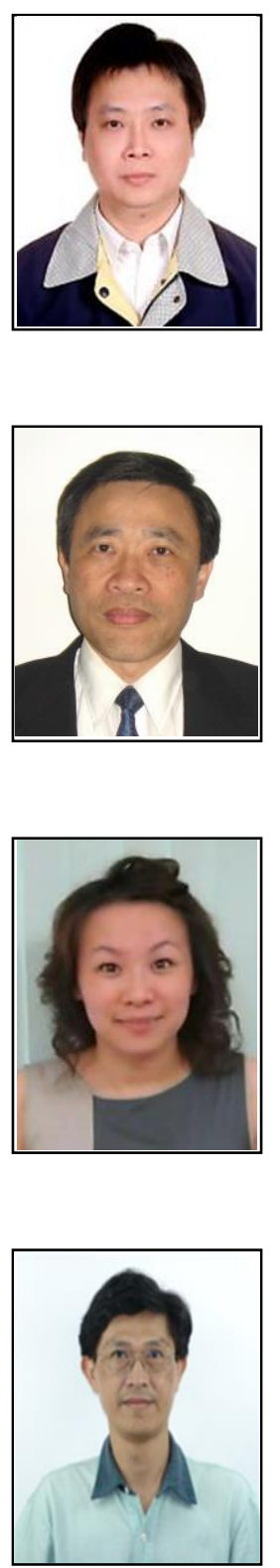

Ful-Chiang $\mathbf{W u}$ is a professor in the Department of Hotel Management at Vanung University, Taiwan. He received his B.S. degree in industrial engineering from Chung-Yuan Christian University and M.S. degree and Ph.D. degree in industrial engineering and management from Yuan-Ze University in Taiwan. His current research activities include robust design, quality management, hotel management and statistics in industrial applications.

Thomas C. Chuang is a professor and the president of Vanung University, Taiwan. He received a Ph.D. degree in engineering from University of California at Los Angles. He has worked for many internationally renowned companies including Eastman Kodak, DuPont and Raytheon in the U.S. His current research interests include tourism management, strategic business planning, pattern recognition, robust design and bibliometric analysis.

Ya-Chi Lee is an assistant professor at Vanung University, Taiwan, in the Department of Hotel Management. She received a M.S degree in urban planning from National Taipei University, and her Ph.D. degree in the College of Management at Yuan-Ze University, Taiwan. Her current research interests include tourism management, international business, quality management and organizational behavior.

Ruei-Lung Lai is a lecturer in the Department of Tourism and Leisure Management at Vanung University, Taiwan. He received his B.S. degree from National Taiwan Ocean University and M.S. degree in computer science from Florida Institute of Technology in the U.S. and Ph.D. degree in electrical engineering from National Taiwan University of Science and Technology in Taiwan. His current research activities include tourism management, quality management, wireless communication management and applications. 